\title{
A Relatively Inexpensive 5.8 Ghz Microwave System for Exploring Electromagnetic Phenomena in Laboratories
}

\author{
Dennis Hite ${ }^{*}$, Colin Murphy, Nagendra Singh \\ Department of Electrical and Computer Engineering, The University of Alabama in Huntsville, Huntsville, Alabama 35899 \\ *Corresponding Author: hitedw@ece.uah.edu
}

Copyright $@ 2014$ Horizon Research Publishing All rights reserved.

\begin{abstract}
This writing describes a $5.8 \mathrm{GHz}$ microwave system that is suitable for use in undergraduate laboratories or as a demonstration tool. The system is constructed mainly from off-the-shelf components and can be configured by anyone possessing a basic knowledge of electronics. The types of demonstrations that the system can be used for is vast and cannot be addressed in a single writing. Therefore, the authors outline one exercise and results that explores wave interference based on propagation and phase delays. The phase delays are produced via spatial and feed line delays.
\end{abstract}

Keywords Electromagnetics Lab, Antenna, Education

\section{Introduction}

Electromagnetism is rooted in many Engineering courses including circuits, electronics, signals/systems, and communications. In the not so far past, wave propagation phenomena could be ignored or lumped into existing theory for most practical situations. However this approach is quickly becoming invalid. This fact was conveyed best in a recent IEEE Transactions on Education correspondence by Clayton Paul: "We are moving into an era where the traditional lumped-circuit analysis of electronic systems will no longer be valid, and electronic systems must be treated as distributed parameter systems. Electromagnetic fields (are) the most important, fundamental subject ...." [1] With the increasing trend of component/system miniaturization, and need for Electromagnetic Compatibility Engineers, it is crucial students being educated in the fields of science and technology achieve a solid foundation in electromagnetics.

Traditionally students struggle in the introductory electromagnetics courses due to its abstract and highly mathematical content. In order to soften the blow of the subject matter many educators are introducing demonstrations and/or lab exercises into their electromagnetics curriculum [2]-[4]. In support of physical experimentation, several off-the-shelf lab apparatus designed to explore electromagnetic concepts have become commercially available. For example products using conductive paper for field tracing and antennas for exploring wave propagation are available from the companies PASCO and Lab-Volt. The ever increasing availability of simulation tools and physical experiments are certainly promising and, based on information from those implementing them, appear to be effective. Effective in both generating student interest and removing some of the abstractness associated with the topic [5], [6]. However prohibitive cost of equipment from commercial sources, i.e. the cost of a single Lab-Volt Antenna measuring system is over \$20,000, makes it difficult for most universities to integrate an electromagnetics lab, or even demonstrations, into their curriculum.

This writing discusses an inexpensive $5.8 \mathrm{GHz}$ microwave system that is comprised of an RF source, RF detector, and set of printed circuit board antennas. The system offers endless possibilities for exploring radiation and propagation of electromagnetic waves. The discussion is limited to one experiment that explores propagating waves and interference suitable as an undergraduate laboratory exercise or classroom demonstration. The finished product is constructed for less than $\$ 1000$ using off-the-self and readily available components, and requires only basic electronics skills to implement.

\section{Components and Configuration}

A block diagram pictured in Fig. 1 represents the completed system. For simplicity of discussion the system is broken down into a transmitting and receiving side. The transmitting side consists of a $5.8 \mathrm{GHz}$ voltage controlled oscillator (VCO) and a wideband $10 \mathrm{~dB}$ amplifier, both available from Mini-Circuits. The VCO and amplifier are connected together, via a SMA-SMA coupler, and together compose the signal source. The output of the amplifier is connected to a 4 element directional array printed on a printed circuit board (PCB), labeled as the Array [7]. All components on the transmitting side are off-the-self, except for the PCB array which is discussed shortly, and require no additional circuitry. 


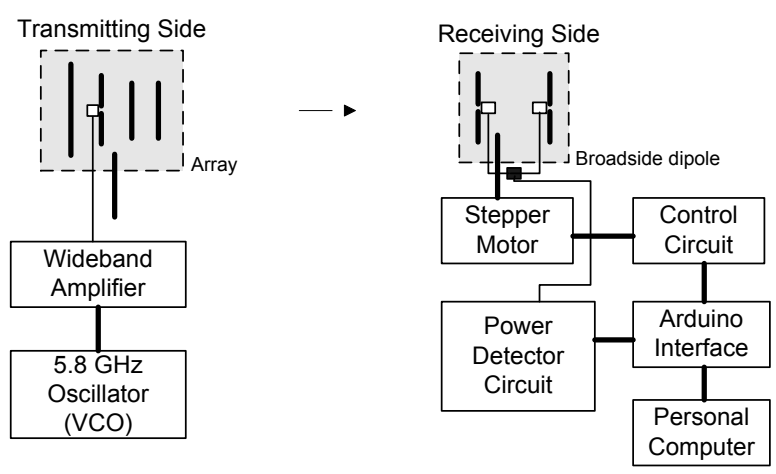

Figure 1. Pictured here is the complete microwave measurement system.

The receiving side consists of a stepper motor, RF power detector, microcontroller (Arduino interface), control circuitry, and a personal computer. These components compose the signal detector which is connected to a broadside dipole array printed on a PCB, labeled the Broadside dipole. Both the transmitting and receiving antennas are attached to pieces of $1 / 2$ inch rigid plastic rod mounted 14 inches above a dove-tail optical rail and connected to the appropriate circuitry via $50 \mathrm{ohm}$ mini coax and SMA connectors, as depicted in Fig. 2. At the antenna side the mini coax is soldered directly to the traces on the PCB. The plastic rods have a 0.2 inch hole drilled 2 inches deep on one end with a set screw for mounting on the shank of the stepper motor. The opposite ends of the rods have a 1 inch depth 1/4-20 tapped hole. On the taped end a 1.5 inch piece of $1 / 4-20$ nylon threaded rod is inserted. The threaded rod provides the attachment point for a $1 / 4-20$ nylon cap nut which holds the PCB antennas in a slot cut the width of a standard hacksaw blade, see Fig. 2. The PCB antennas can be secured to the cap nuts with a small amount of epoxy.
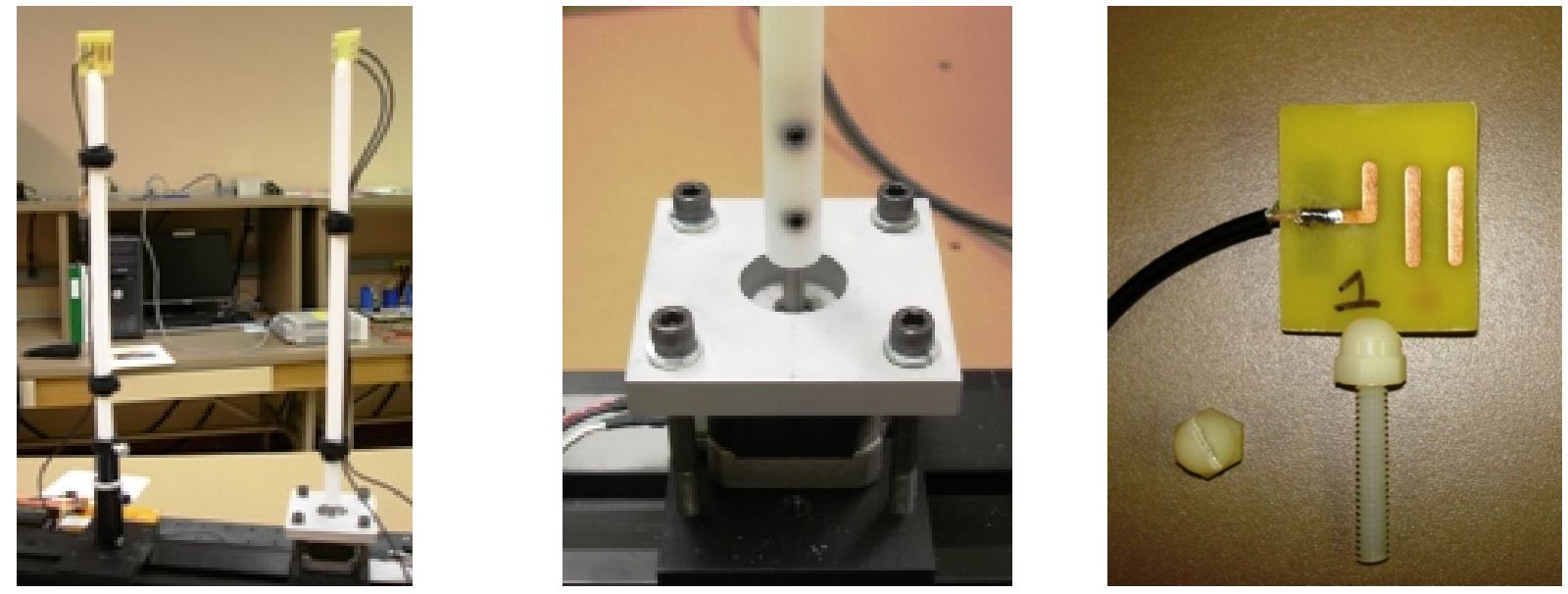

Figure 2. Shown here are the antenna mounting details for the system.

The antenna parameters were optimized through trial and error. The initial antenna dimensions were guided by the work of Navarro et al [8]. Several different antennas where analyzed by varying parameters for element length and spacing. The final parameters for the antennas displaying the best performance are defined in Fig. 3.
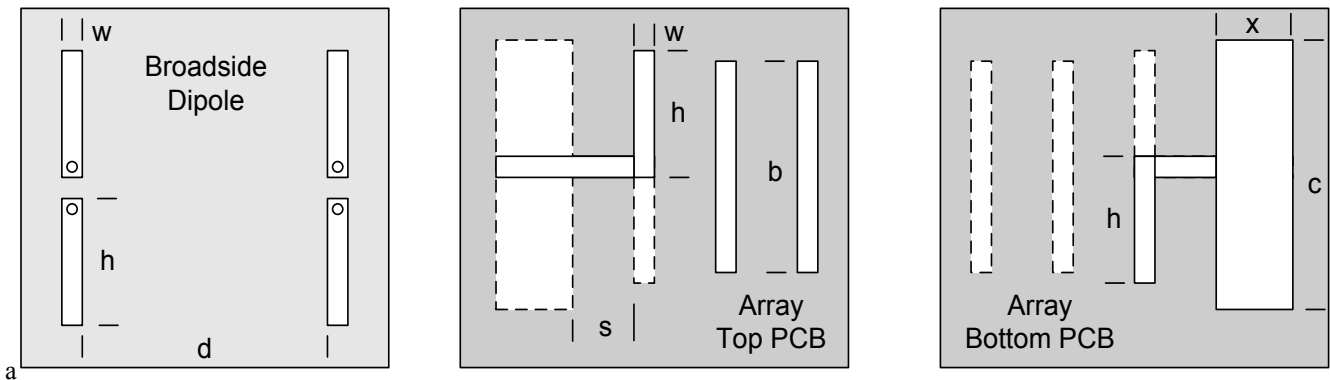

Figure 3. PCB Antennas used for the interference exercise. The Broadside dipole is 1 single sided PCB, while the Array is 1-double sided PCB.

It should be apparent in Fig. 3 that the Broadside dipole is printed on a single sided PCB, while the Array requires use of 2 sides [9]. For the Broadside dipole the element lengths (h) are $9 \mathrm{~mm}$, the element spacing (d) is $18 \mathrm{~mm}$, and the trace width (w) equal to $2 \mathrm{~mm}$. The Array driver element length (h) is $9 \mathrm{~mm}$. The reflector element length (c) is $18 \mathrm{~mm}$, the two separate director elements (b) is $15 \mathrm{~mm}$, for a total of two directors. Element spacing (s) is $4 \mathrm{~mm}$ and trace width (x) is equal to $5 \mathrm{~mm}$. All dimensions are based on the VCO tuned to a signal frequency equal to $5.75 \mathrm{GHz}$. 
The stepper motor provides for rotation of the Broadside dipole (receiving antenna). The power detector, by design, outputs a voltage that is proportional to the amplitude of RF detected. Both the stepper motor and power detector is available off-the-shelf from the vendors listed in the parts list available from the authors.

The power detector circuit, control circuit, and Arduino platform provide control and data acquisition interface for the system. The Arduino platform is an open source programmable microcontroller that includes several Analog/Digital inputs and outputs. Four of the digital outputs are used to drive the stepper motor via the controller circuit pictured in Fig. 4.

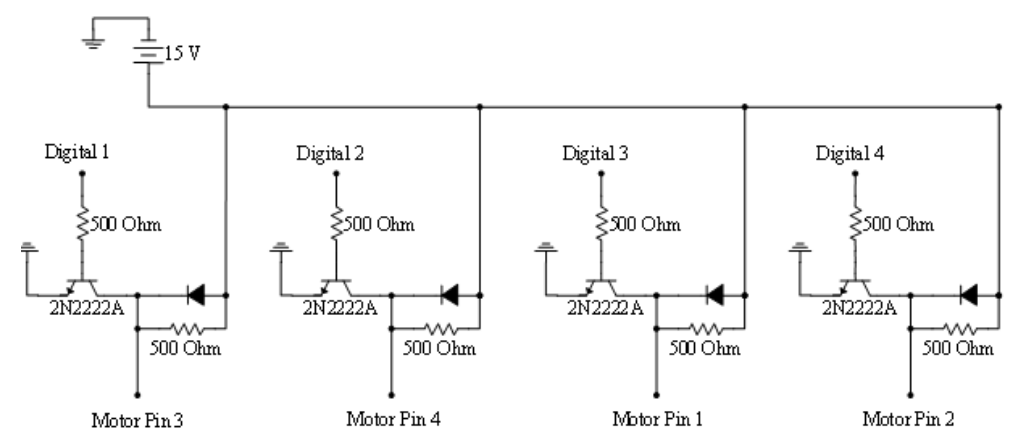

Figure 4. The schematic of the stepper motor control circuit that interfaces the Arduino and stepper motor

One of the on-board analog input channels is used to receive data from the RF power detector via the power detector circuit outlined in Fig. 5. The motor control circuitry and power detector circuit are not off-the-shelf and must be constructed in house. Most of the components can be found in any electronics shop or ordered online through a vendor such as Digi-Key Corporation. The exception is the INA126 instrumentation amplifier which can be ordered through Texas Instruments or one of their distributors.

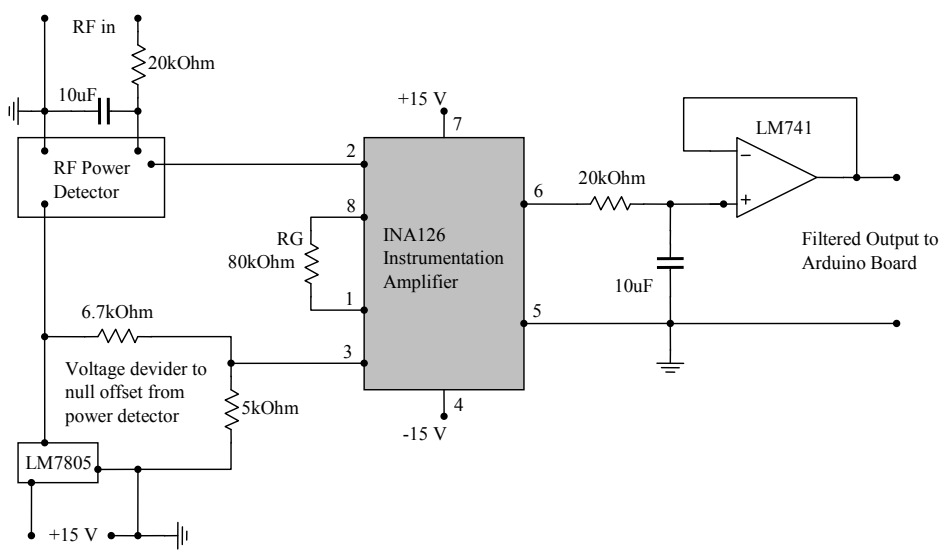

Figure 5. The schematic of the power detector and required circuitry.

The Arduino platform interfaces with a personal computer via USB. The USB interface is used to load the programs the Arduino runs. It is also the path used to pass acquired data to MATLAB on the PC. A program that controls the stepper motor is available from the authors. Based on the provided Arduino code and the stepper motor specified in the parts list, the appropriated connections between the two are; Arduino Digital pin 3 to stepper motor pin 1, Digital pin 5 to stepper motor pin 4, Digital pin 6 to stepper motor pin 3, and Digital pin 9 to stepper motor pin 6. Due to current switching used to drive the stepper motor it is important that an independent power supply is used that no other components of the system uses as a power source.

The power detector circuitry provides signal conditioning of the raw output received from the RF power detector. It is comprised of; 1) two low pass filters to reduce signal noise, 2) an instrumentation amplifier to remove the dc offset produced by the RF power detector while amplifying the received signal. The necessary MATLAB code is provided by the authors via email. The code establishes communication, runs the Arduino program, records received data, and generates plots of the data. Based on the MATLAB program the appropriated connections are; the Filtered output connected to Arduino Analog pin 0 and ground. 


\section{Experiment}

The information contained in Section III assumes all components used are from the parts list and programs available from the authors via email.

\subsection{Initial Setup}

The RF transmitter (VCO) must be set to a specific voltage at Vtune to resonate at the pre-determined frequency of 5.75 $\mathrm{GHz}$. Connect the appropriate voltage supplies to the VCO and set the voltage at Vtune initially to zero volts. Connect a coaxial cable from the output of the VCO to the input of a spectrum analyzer with appropriate attenuation as needed. Slowly adjust the voltage at Vtune until the output on the spectrum analyzer reads $5.75 \mathrm{GHz}$. Record this voltage for future use, this is the voltage needed at Vtune to ensure a $5.75 \mathrm{GHz}$ output signal.

The nature of the experimental apparatus (system) makes it susceptible to RF reflections and multi-path effects. Therefore it is important that attention is paid to the following setup details which help reduce the effects.

To begin connect the appropriate power supplies to the Arduino, op amp circuitry, RF power detector, and the VCO. Be sure that Vtune on the VCO is set to the pre-determined voltage producing a $5.75 \mathrm{GHz}$ output signal. Do not power on the VCO until it has been connected to an antenna or properly terminated into a $50 \mathrm{ohm}$ load. Next connect the Arduino to a computer via USB and connect an oscilloscope to the output of the op amp circuitry just before input to the Arduino. The oscilloscope connection will be used to monitor the raw analog signal from the RF power detector. Remember the stepper motor requires an independent power source.

Next connect the array antenna to the VCO. Mount the array on the non-rotating plastic rod. Then aim the array directly at the rotating rod, where the Broadside dipole will be mounted. Connect the input of the RF power detector to a SMA T-coupler and the Broadside dipole. Mount and center the Broadside dipole on the rotating shaft, making sure that the center, halfway between the two dipoles, is positioned correctly. Rotate the Broadside dipole, relative to the side without wires, so that the right edge of the PCB is pointing towards the transmitting (Array) antenna. This is important as the motor will rotate clockwise and over the course of its rotation expose the front to the transmitting antenna. Initially position the Array antenna about $15 \mathrm{~cm}$ away from the Broadside dipole. This completes the initial setup the data collection details follow.

\subsection{Collecting Data}

Disconnect one of the dipoles on the Broadside, with one dipole inactive a base line plot, with no interference pattern, can be produced. Turn on all power sources at this stage. Open MATLAB and run the rotation180 program, this initiates a 180 degree rotation of the Broadside dipole antenna, then returns to its original position. During this time MATLAB will acquire data and you will be prompted to generate a plot. The plot will represent the radiation pattern of a single vertical dipole antenna. Note any data/plots the user wishes to save must be done before the next execution of the program. By default the MATLAB program overwrites the previous data. When MATLAB calls the plot function, the array will rotate and return once more to its original position. This is simply a response by the Arduino to any sensed input via the USB cable and no additional data is collected.

Reconnect both dipoles of the Broadside dipole to the $\mathrm{T}$-coupler. Due to the effects of multi-path a positional RF null must be found in order to observe the Broadsides radiation pattern without interference from reflections in a closed environment. While monitoring the oscilloscope, slowly push the transmitting antenna (Array) along the rail until the voltage on the oscilloscope reads zero (or as close to zero as possible) then secure it to the rail. This adjustment may require a few attempts to produce the desired results. Once a suitable position is found run the rotation 180 program to acquire data. Once again you will be prompted to generate a plot. The plot will represent the radiation pattern of a Broadside vertical dipole antenna.

For the final data run connect a one half wavelength coaxial snub, we found the optimal length to be $62 \mathrm{~mm}$ by trail and error, between one of the Broadside dipoles and the T-coupler. This connection introduces a one half wavelength feed line delay, a.k.a. excitation phase delay, to one element of the Broadside dipole array. The resulting radiation pattern will be that of an end fire array. This find the null of the broadside of the dipole array, then return the array position to the 0 deg element facing the array. Run the rotation 180 program to acquire data. Once again you will be prompted to generate a plot. The plot will represent the radiation pattern of a Broadside vertical dipole antenna with an additional feed line delay of 180 degrees.

There should now exist data and plots for three different configurations; single vertical dipole, broadside array dipole with element spacing equal to one half wavelength, and broadside array dipole with element spacing equal to one half wavelength and a half wavelength feed line delay.

\section{Results}

For both cases the students should be encouraged to sketch and discuss the observed signal interference patterns and explain both conceptually and mathematically the interactions based on their observations and measurements. Specifically, the effects resulting from element spacing and transmission line delays (a.k.a. excitation phase) compared to a single dipole radiation pattern.

Antennas are easy to analyze in spherical coordinates ( $r, \theta$, $\varphi$ ), where the angles $\varphi$ and $\theta$ are measured from the $\mathrm{x}$ and $\mathrm{z}$ axes of the Cartesian co-ordinate system, respectively. In our set up we assume that the two dipoles are symmetrically 
placed around the origin and aligned along the $\mathrm{x}$ axis. They are oriented parallel to the $\mathrm{z}$ axis as pictured in Fig 6 .

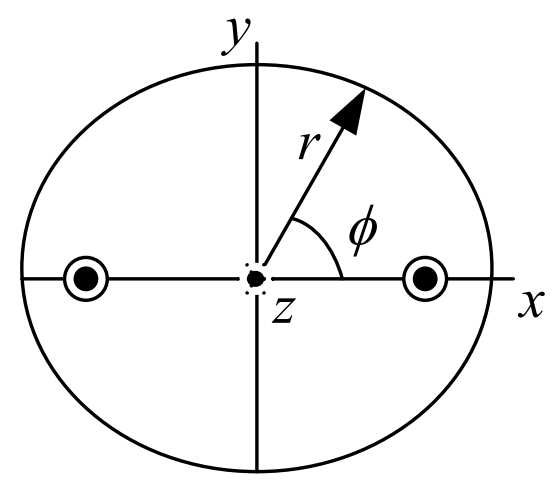

Figure 6. Here the rotation angle $\phi$ with respect to the dipole orientation, into the page, is shown, while $\theta$ is taken from the positive $z$ axis.

We first discuss the radiation pattern of one element of the array, which is later used as a basis for discussing the pattern of the array. Fig. 7 shows the radiation patterns as functions of $\varphi$ and $\theta$, respectively. As expected, Fig. 7.A shows the pattern is nearly circular in the $x-y$ plane at $\theta=\pi / 2$. The pattern in Figure 7.B closely approximates $\sin \theta$. The measured patterns and their comparison with that expected from theory establish that the antennas constructed by us closely radiate like dipoles.
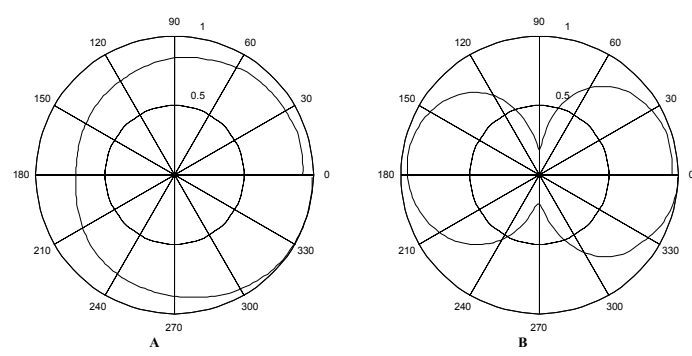

Figure 7. The radiation pattern of a single dipole on the Broadside array. First in $\mathrm{A}$ as a function of $\phi$ then $\theta$ in $\mathrm{B}$.

Next we examine the radiation pattern of the two-element array with $\theta=\pi / 2$. The expected field intensity patterns are computed using standard array antenna theory. The theory predicts in the far field, assuming zero coupling between individual elements, the total field can be computed through vector addition of the fields generated by the individual elements in the array [10]. The equation for this computation is

$\overline{E_{t}}=\hat{a}_{\theta} j \eta \frac{k I_{0} l e^{-j k r}}{4 \pi r} \sin (\theta)\left\{2 \cos \left[\frac{1}{2}(k d \cos (\phi)+\beta)\right]\right\}$.

Equation (1.1) can be divided into the product of two terms. The first term represents the field generated by a single array element,

$$
\hat{a}_{\theta} j \eta \frac{k I_{0} l e^{-j k r}}{4 \pi r} \sin (\theta) .
$$

The second term

$$
2 \cos \left[\frac{1}{2}(k d \cos (\phi)+\beta)\right],
$$

is commonly referred to as the Array Factor (AF). ${ }^{11}$ For this exercise the first term (1.2) is set to unity, by assuming equal excitation amplitudes on all elements, and setting $\theta$ equal $\pi / 2$ to since the rotation is only carried out with respect to $\phi$. The factor of 2 on the remaining term is dropped to create a normalized curve. The term $k$ is the wave number $2 \pi / \lambda, d$ is the element spacing in terms of wavelength, and $\beta$ is the excitation phase difference between the elements.

For the Broadside array configuration, $d=\lambda / 2$ and $\beta=0$, the theoretical curve is computed for $0 \leq \phi \leq \pi$ and plotted along with the experimental data in Fig. 8. It is important to comment that he shape of the expected curve fits well with the computed curve, although the areas under the curves do not match. This situation occurs due to the fact the coaxial connections on the experimental Broadside array directs more power toward the front lobe than the rear lobe. The computed curve divides the power equally between the front and rear lobes.

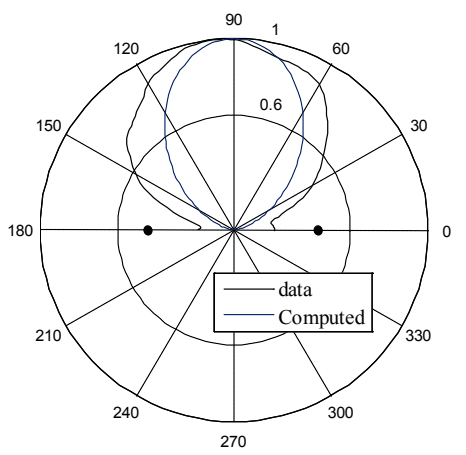

Figure 8. Normalized Polar plot of computed and experimental data for the Broadside array with $d=\lambda / 2$ and $\beta=0$.

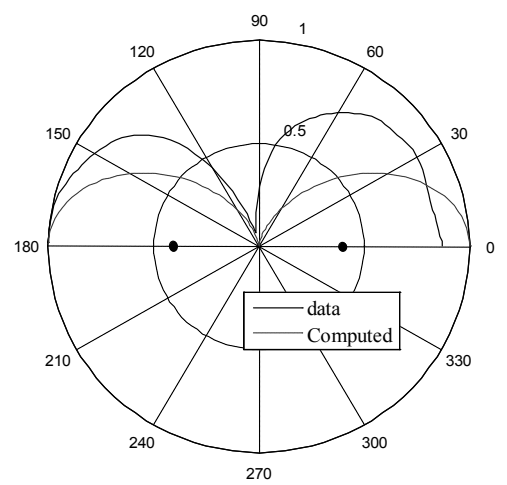

Figure 9. Polar plot of computed and experimental data for the Broadside array with $d=\lambda / 2$ and $\beta=\pi$, producing end fire from excitation phase difference. 
For the Broadside array as an end fire antenna, $d=\lambda / 2$ and $\beta=\pi$, the theoretical curve is computed for $0 \leq \phi \leq \pi$ and plotted along with the experimental data in Fig. 9. Here again we comment the shape of the power distribution is correct, ignoring the non symmetries created by the physical antenna connections.

\section{Concluding Remarks}

In this writing we have presented a simple, affordable, microwave system and accompany wave interference experiment. For comparison we performed a literature search on the Lab-Volt Antenna Training and Measuring System. As a result we found several examples of generated plots, and general system information. The literature suggests that the system from Lab-Volt has several features our system does not. The system from Lab-Volt has the ability to produce three dimensional plots and plot the radiated magnetic, as well as, the electric field components all being in color. The Lab-Volt system also comes with several additional antennas such as horns, helical, and slotted-waveguides. Of course all the additional features come at a cost; we believe our system delivers reasonable accurate results suitable and affordable for most undergraduate laboratory and demonstrations. Our goal is to motivate other educators to include laboratory exercises as part of their Electromagnetics curriculum. The end result being students will enter the work force possessing a better understanding of the material and prepared to meet existing and future challenges.

\section{REFERENCES}

[1] C. R. Paul, "Areas of Electrical and Computer Engineering Education That Require Increased Emphasis," IEEE Transactions on Education, 52, (1), 200-201, (2009).

[2] A. D. Kipple "Learner-centered laboratory activities for an undergraduate electromagnetics course," Antennas and Propagation Society International Symposium, 2007 IEEE, 5375-5378, (2007).

[3] M. A. Jensen, R. H. Selfridge, K. F. Warnick, "System-level microwave design projects," Antennas and Propagation Magazine, IEEE, 43, (5), 138-142, (2001).

[4] J. L. Drewniak, T. H. Hubing, T. P. Van Doren, Sha Fei, "Integrating electromagnetic compatibility laboratory exercises into undergraduate electromagnetics," Electromagnetic Compatibility 1995. Symposiu Record. IEEE International Symposium, 35-40, 14-18, (1995).

[5] F. T. Ulaby, B. L. Hauck, "Undergraduate electromagnetics laboratory: An invaluable part of the learning process," Proceedings of the IEEE , 88, (1), 55-62, (2000).

[6] M. Popovic, D. D. Giannacopoulos, "Assessment-based use of CAD tools in electromagnetic field courses," IEEE Transactions on Magnetics , 41, (5), 1824-1827, (2005).

[7] All the antennas are fabricated on PCBs, standard FR4 0.062 inch thick board material.

[8] E. A. Navarro, J. M. Blanes, J. A. Carrasco, C. Reig, "Yagi-Like Printed Antennas for Wireless Sensor Networks," Sensor Technologies and Applications, SensorComm 2007, International Conference on Sensor Technologies and Applications, 254-259, 14-20 (2007).

[9] It is important to inform the PCB manufacturer that the only copper left on the PCB after processing is that specified by the antenna traces.

[10] Constantine A. Balanis, Antenna Theory Analysis and Design, 3rd ed. (Wiley and Sons, Hoboken, New Jersey, 2005), pp 283-300. 